\title{
AVALIAÇÃO DO TREINAMENTO DE OPERADORES DE HARVESTER COM USO DE SIMULADOR DE REALIDADE VIRTUAL ${ }^{1}$
}

Eduardo da Silva Lopes² ${ }^{2}$ Enerson Cruziniani ${ }^{3}$, Antonio José de Araujo ${ }^{2}$ e Paulo Candido da Silva ${ }^{4}$

\begin{abstract}
RESUMO - Este trabalho teve por objetivo avaliar os potenciais ganhos de operadores de harvester no treinamento com uso de simuladores de realidade virtual. Foram coletados dados durante o treinamento de 39 operadores, avaliando-se os seus desempenhos nas variáveis: tempo de execução no corte da árvore, direção de queda, altura de corte, eficiência no processamento, eficiência no empilhamento e produtividade. Os resultados permitiram a conclusão de que o simulador de realidade virtual é uma ferramenta viável do ponto de vista técnico e essencial no apoio ao treinamento de operadores de máquinas florestais, possibilitando a obtenção de elevados ganhos de qualidade e produtividade. O melhor desempenho dos operadores ocorreu com a variável produtividade, cujo ganho médio foi de $41,3 \%$ em relação ao início do treinamento, seguido pelo tempo de execução, com $38,8 \%$; e pela direção de queda, com ganho médio de $36,1 \%$.
\end{abstract}

Palavras-chave: Treinamento, simulador e produtividade.

\section{EVALUATION OF HARVESTER OPERATOR TRAINING USING VIRTUAL REALITY SIMULATORS}

\begin{abstract}
The present work aimed to evaluate the potential gains from the use off virtual reality simulators in the training of harvester operators. Data were collected during the training of 39 operators, regarding their performance in the following variables: time of execution, fall direction, cut height, processing efficiency and piling up and productivity. The results led to conclude that virtual reality simulators are viable and essential tools in the training of forest machine operators, increasing quality and productivity. Productivity presented the best operator performance with gains of $41.3 \%$ in relation to the beginning of the training, followed by time of execution with $38.8 \%$ and fall direction, with a mean gain of $36.1 \%$.
\end{abstract}

Keywords: Training, simulator and productivity.

\section{INTRODUÇÃO}

As atividades de colheita da madeira são de grande importância no setor florestal, principalmente em termos econômicos, pois representa em torno de $50 \%$ ou mais do custo final da madeira posta na fábrica (MACHADO, 2002). Além disso, deve-se considerar a complexidade dessas atividades, dado o grande número de variáveis que afetam a produtividade e, conseqüentemente, os custos operacionais (CANTO et al., 2006).

Segundo Moreira (2004), a mecanização da colheita florestal em nosso país intensificou-se significativamente a partir da década de 1990, com a abertura do mercado pelo governo brasileiro à importação de máquinas e equipamentos de países de maior tradição florestal.

\footnotetext{
${ }^{1}$ Recebido em 27.02.2007 e aceito para publicação em 20.02.2008.

${ }^{2}$ Departamento de Engenharia Florestal da UNICENTRO, Irati, PR. E-mail: <eslopes@irati.unicentro.br; dearaujo@ irati.unicentro.br>.

${ }^{3}$ Curso de Engenharia Florestal da UNICENTRO, Irati, PR. E-mail: <enersoncruziniani@ @otmail.com>.

${ }^{4}$ Centro de Formação de Operadores Florestais (CENFOR) da UNICENTRO, Irati, PR. E-mail: <paulocandido@irati.unicentro.br>.
} 
Além disso, outros fatores contribuíram para a mecanização, como o aumento da produtividade das florestas e dos custos de mão-de-obra e a necessidade de executar o trabalho com maior segurança operacional e de redução nos custos de produção. Essas circunstâncias levaram empresas brasileiras a passarem da colheita manual ou semi-mecanizada para sistemas totalmente mecanizados, com máquinas de alta tecnologia, produtividade e elevados custos. Por isso, a realização de estudos que aperfeiçoem as operações e reduzam os custos operacionais torna-se cada vez mais importante (BRAMUCCI e SEIXAS, 2002).

Atualmente existem, nas empresas florestais, elevado número de máquinas e equipamentos de colheita de madeira de alta tecnologia que exigem cada vez mais operadores capacitados. A escassez desses operadores tem provocado muitos danos às máquinas, comprometendo a produtividade e a qualidade do trabalho e aumentado os custos de produção, além de impactos ao meio ambiente (PARISE e MALINOVSKI, 2002). Segundo Packalén (2001), o treinamento de operadores de máquinas de colheita florestal apresenta elevado custo quando comparado com o de profissionais de outros setores. Com o alto nível tecnológico das atuais máquinas florestais, não é técnica e economicamente viável a realização de treinamentos em situação real.

Em diversos sistemas de toras curas, o harvester é a máquina principal, utilizada na derrubada e processamento da madeira e, em alguns casos, no descascamento das árvores, no desgalhamento e no corte em toras de comprimento predeterminado, deixando as toras grupadas e prontas para serem retiradas da área. Trata-se de um equipamento autopropelido, constituído por um conjunto automotriz de alta mobilidade dentro da floresta e de boa estabilidade, um braço hidráulico e um cabeçote processador (AMABILINI, 1991).

No momento, vêm sendo desenvolvidas técnicas que visem melhorar o desempenho de máquinas e operadores, a fim de maximizar a produtividade. Para Lacerda e Mazon (2002), a forma mais avançada de treinamento eficiente e disponível no momento é o simulador de realidade virtual, em que o usuário pode realizar a imersão, navegação e interação em um ambiente sintético tridimensional gerado por computadores.
Estudos realizados na Suécia indicaram que o uso de simuladores de realidade virtual propiciou um aprendizado eficiente para os futuros operadores de máquinas, reduzindo significativamente os custos de treinamento. Foi comparado o desempenho de dois grupos de treinandos, em que o primeiro recebeu treinamento de $10 \mathrm{~h}$ no simulador virtual e $20 \mathrm{~h}$ na máquina, enquanto o segundo grupo recebeu treinamento de 100 h na máquina, em situação real de campo. Em seguida, ambos os grupos foram reavaliados, e os resultados indicaram que ambos os grupos alcançaram o mesmo rendimento e qualidade do trabalho (PARISE e MALINOVSKI, 2002).

Este trabalho teve por objetivos avaliar o desempenho de operadores de harvester e quantificar potenciais ganhos durante o treinamento com o uso de simuladores de realidade virtual.

\section{MATERIAL E MÉTODOS}

\section{1. Área de Estudo}

O trabalho foi conduzido no Centro de Formação de Operadores Florestais (CENFOR) do Departamento de Engenharia Florestal da Universidade Estadual do Centro-Oeste (UNICENTRO), em Irati, Paraná.

\subsection{População e Amostragem}

A população pesquisada foi composta por trabalhadores de diversas empresas florestais que receberam treinamento em simulador de realidade virtual, com carga horária de $40 \mathrm{~h}$. Os dados foram coletados em uma amostra de 39 trabalhadores florestais, sem experiência na operação de harvester, no período de agosto de 2005 a outubro de 2006.

\subsection{Equipamentos Utilizados}

Foram utilizados simuladores de realidade virtual, montados em computador, pertencentes ao CENFOR, utilizando-se o programa de simulação harvester, da Simlog Simulation Launcher.

\subsection{Metas de Desempenho Estabelecidas no Treinamento}

Foram estabelecidas metas de desempenho (Tabela 1) para os operadores em cada variável nos diferentes módulos operacionais, cujo propósito era estimular os operadores a alcançar e superar um desempenho satisfatório ao final do treinamento. 
Tabela 1 - Metas de desempenho estabelecidas no treinamento Table 1 -Established goals of performance in the training

\begin{tabular}{lccc}
\hline \multicolumn{1}{c}{ Variável } & & \multicolumn{2}{c}{ Módulos } \\
\cline { 2 - 4 } & II & III & VI \\
\hline Tempo de execução (s) & 25 & 25 & 90 \\
Direção de queda (graus) & 6 & 6 & - \\
Altura de corte (cm) & 5 & 10 & 10 \\
Eficiência no processamento (\%) & - & - & 95 \\
Eficiência no empilhamento (\%) & - & - & 98 \\
Produtividade $\left(\mathrm{m}^{3} / \mathrm{h}\right)$ & - & - & 92 \\
\hline
\end{tabular}

\subsection{Módulos Operacionais Avaliados}

O treinamento dos operadores de harvester no simulador de realidade virtual era composto por sete módulos operacionais, conforme descrição a seguir. O módulo I não foi avaliado, pois se referia apenas à identificação de comandos dos joysticks.

a) Módulo II - Controle de movimentos do cabeçote: Controlava os movimentos do braço e cabeçote, sem realização da derrubada da árvore, com duração de $6 \mathrm{~h}$.

b) Módulo III - Execução de derrubada simples: Executava a derrubada de uma única árvore na direção de queda desejada, controlando a altura de toco, com duração de $8 \mathrm{~h}$.

c) Módulo IV - Execução de derrubada simples e processamento: Executava a derrubada direcional e processamento de uma árvore, controlando manualmente o comprimento da tora e altura do toco, com duração de $6 \mathrm{~h}$.

d) Módulo V - Execução de derrubada simples e empilhamento: Executava a derrubada, processamento e empilhamento da árvore em alvos específicos, com duração de 6 h.

e) Módulo VI - Execução de derrubada múltipla e empilhamento: Executava a derrubada e processamento de grupos de árvores, controlando os comprimentos e empilhando as toras em alvos específicos, com duração de $4 \mathrm{~h}$.

f) Módulo VII - Processamento: Executava o processamento de árvores dispostas na beira do talhão, empilhando as toras nos alvos específicos, com duração média de $8 \mathrm{~h}$.

\subsection{Variáveis Analisadas}

O treinamento no simulador compreendeu sete módulos operacionais, em que foram avaliadas todas as variáveis pertinentes a cada módulo. O desempenho do operador para cada variável foi gerado pelo próprio software do simulador. Na Tabela 2 é mostrado as variáveis contempladas em cada módulo, com a respectiva descrição.

\subsection{Coleta e Análise de Dados}

Os dados foram coletados no decorrer do treinamento em três períodos cronológicos distintos, a partir da média de 10 sessões realizadas no início, meio e fim de cada módulo. Considerando que cada módulo contemplava diferentes variáveis, elas foram avaliadas independentemente.

Para melhor caracterizar o desempenho obtido pelos operadores no treinamento, avaliaram-se somente os módulos II, III, VI e VII, que melhor representa as operações de derrubada e processamento com o harvester.

Para a obtenção do desempenho médio obtido pelos operadores nos treinamentos, foi utilizada a seguinte expressão:

$$
g(\%)=\left(1-\left(\frac{m_{2}}{m_{1}}\right)\right) .100
$$

em que: $\mathrm{g}(\%)=$ ganho porcentual em relação ao início do treinamento na respectiva variável e módulo; $\mathrm{m}_{2}$ $=$ média no final do módulo; e $\mathrm{m}_{1}=$ média no início do módulo.

Os valores obtidos durante os treinamentos foram analisados a partir da plotagem de gráficos, análise estatística e teste de Tukey a 5\% de probabilidade.

R. Árvore, Viçosa-MG, v.32, n.2, p.291-298, 2008 
Tabela 2 - Descrição das variáveis analisadas nos diferentes módulos

Table 2 -Description of the variables analyzed in the different modules

\begin{tabular}{|c|c|c|c|c|c|c|}
\hline \multirow[t]{2}{*}{ Variável } & \multirow[t]{2}{*}{ Descrição } & \multicolumn{5}{|c|}{ Módulo } \\
\hline & & II III & IV & $\mathrm{V}$ & VI & VII \\
\hline $\begin{array}{l}\text { Tempo de execução } \\
(\mathrm{s})\end{array}$ & $\begin{array}{l}\text { Expressa o tempo, em segundos, em que o operador } \\
\text { realizou o ciclo desejado para cada módulo. }\end{array}$ & $\sqrt{ } \quad \sqrt{ }$ & $\sqrt{ }$ & $\sqrt{ }$ & $\sqrt{ }$ & $\sqrt{ }$ \\
\hline $\begin{array}{l}\text { Direção de queda } \\
\text { (graus) }\end{array}$ & $\begin{array}{l}\text { Mostra a orientação de queda da árvore em graus, } \\
\text { em relação à direção desejada, na derrubada da árvore. }\end{array}$ & $\sqrt{ }$ & & & & \\
\hline $\begin{array}{l}\text { Altura de corte } \\
(\mathrm{cm})\end{array}$ & $\begin{array}{l}\text { Refere-se à altura de corte em relação à posição } \\
\text { desejada. Pode ser expressa em relação à altura do toco } \\
\text { ou, mesmo, em relação à altura de uma marca } \\
\text { pré-determinada ao longo da árvore. }\end{array}$ & & $\sqrt{ }$ & $\sqrt{ }$ & $\sqrt{ }$ & \\
\hline $\begin{array}{c}\text { Eficiência no } \\
\text { processamento }(\%)\end{array}$ & $\begin{array}{l}\text { Mede a quantidade porcentual das árvores efetivamente } \\
\text { processadas durante os ciclos. }\end{array}$ & & $\sqrt{ }$ & $\sqrt{ }$ & $\sqrt{ }$ & $\sqrt{ }$ \\
\hline $\begin{array}{c}\text { Eficiência no } \\
\text { empilhamento }(\%)\end{array}$ & $\begin{array}{l}\text { Mede a porcentagem de toras depositadas corretamente } \\
\text { dentro da caixa de empilhamento, determinada no } \\
\text { módulo da simulação. }\end{array}$ & & & $\sqrt{ }$ & $\sqrt{ }$ & $\sqrt{ }$ \\
\hline $\begin{array}{l}\text { Produtividade } \\
\left(\mathrm{m}^{3} / \mathrm{h}\right)\end{array}$ & Expressa a produtividade efetiva obtida na operação completa. & & & & $\sqrt{ }$ & $\sqrt{ }$ \\
\hline
\end{tabular}

\section{RESULTADOS E DISCUSSÃO}

\subsection{Variáveis Analisadas}

\subsubsection{Tempo de Execução}

No decorrer dos treinamentos foi possível observar que o tempo de execução influenciou diretamente a qualidade e produtividade. Na Figura 1, apresentam-se os tempos médios consumidos pelos operadores na execução da operação. Como pode ser visto nos módulos II e III, o tempo de execução foi baixo em virtude de se tratar de um módulo inicial, em que o operador realizava somente parte da operação, enquanto nos módulos VI e VII, o tempo de execução era superior, por se tratar de uma operação completa.

Como pode ser observado, houve significativa evolução no desempenho dos operadores no decorrer do treinamento, cujos ganhos porcentuais ao final de cada módulo foram de $52 \%, 34,4 \%, 25 \%$ e 33,4\% nos módulos II, III, VI e VII, respectivamente.

É importante enfatizar a significativa evolução no desempenho dos operadores $(52,0 \%)$, no início e durante a execução do módulo II, ocasião em que os operadores estavam familiarizando-se com os comandos e movimentos da máquina. No módulo III foi possível verificar, também, uma evolução no desempenho dos operadores, e 56,4\% dos indivíduos avaliados atingiram a meta estabelecida de $25 \mathrm{seg}$. Em relação ao módulo VI, os ganhos de desempenho foram de apenas $25 \%$, pois os operadores já haviam adquirido experiência e habilidade nos módulos anteriores.

R. Árvore, Viçosa-MG, v.32, n.2, p.291-298, 2008

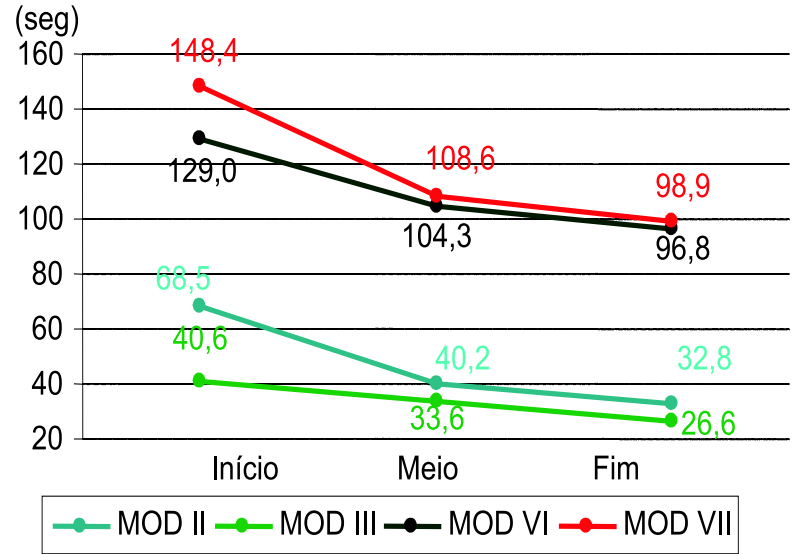

Figura 1 - Tempo médio de execução. Figure 1-Mean execution time.

Por fim, o módulo VII, que tratava somente do processamento da madeira na beira da estrada, o ganho de desempenho dos operadores foi de 33,4\%. Foi possível, ainda, observar maiores dificuldades dos operadores em apanhar as árvores para a realização do processamento.

\subsubsection{Direção de Queda}

A direção de queda foi a variável, que os operadores tiveram maior dificuldade de executar. Foi possível observar que eles dificilmente atingiam a meta estabelecida, devido às exigências de domínio da grua e cabeçote na operação de derrubada. 


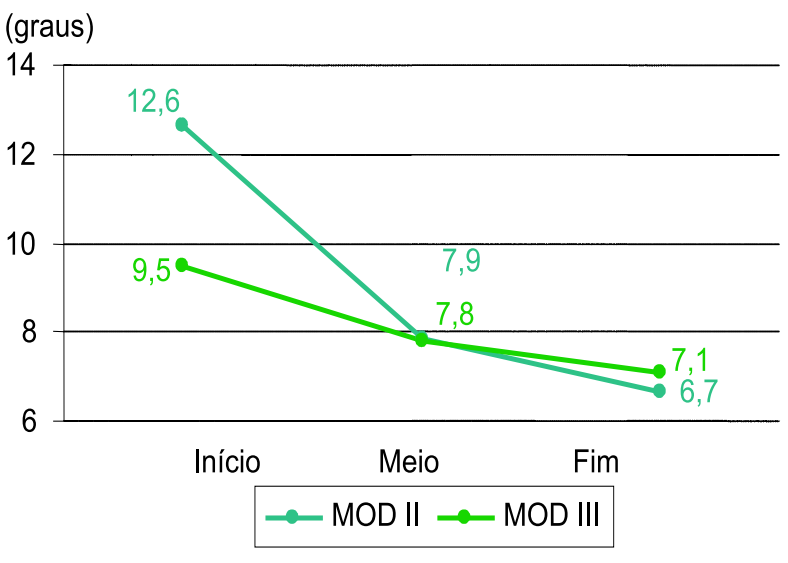

Figura 2 - Valores médios da variável direção de queda de execução dos módulos II e III.

Figure 2-Mean values for the variable fall direction in modules II and III.

Os valores médios de direção de queda obtidos pelos operadores no início, meio e fím do módulo II foram de 12,6; 7,9; e 6,7 graus, respectivamente, com ganho médio de $47,1 \%$. No módulo III, observou-se evolução no desempenho dos operadores somente no início, ficando os resultados nos demais momentos do treinamento semelhantes entre si. Os valores médios de direção de queda foram de 9,$5 ; 7,8$; e 7,1 graus, no início, meio e fim do treinamento, respectivamente, com ganho médio de 25,2\% (Figura 2).

\subsubsection{Altura de Corte}

A altura de corte foi considerada uma variável de maior importância na avaliação do desempenho dos operadores, principalmente no módulo II, que exige coordenação motora do operador na realização dos cortes em diferentes posições, além de ser limitante na qualidade da operação e na identificação de erros cometidos. Nos demais módulos, a altura de corte ficou limitada ao posicionamento do cabeçote próximo ao solo, sendo o ganho médio de desempenho obtido pelos operadores na ordem de 49,4\% (Figura3).

\subsubsection{Eficiência no Processamento}

Os operadores obtiveram, de modo geral, desempenho satisfatório em relação a essa variável, e os índices obtidos estavam próximos das metas estabelecidas. Como pode ser visto na Figura 4, o desempenho médio dos operadores nos módulos VI e VII foi de 90,6 e 91,7\%, respectivamente, enquanto no módulo VI 66,7\% dos operadores obtiveram índices acima da meta estabelecida. Já o módulo VII, em que o operador praticava apenas o processamento das árvores, os valores médios de desempenho comportaramse de forma satisfatória, com valores próximos às metas estabelecidas.

\subsubsection{Eficiência no Empilhamento}

A eficiência no empilhamento é considerada outra variável importante na avaliação do desempenho dos operadores, exigindo elevada coordenação motora e noção espacial dos operadores para a execução da tarefa. Como pode ser visto na Figura 5. O módulo VI, que simula a operação completa e exige domínio completo do cabeçote, o desempenho médio obtido pelos operadores foi de 95,4; 97,8; e 97,8\% no início, meio e fim do módulo, respectivamente.

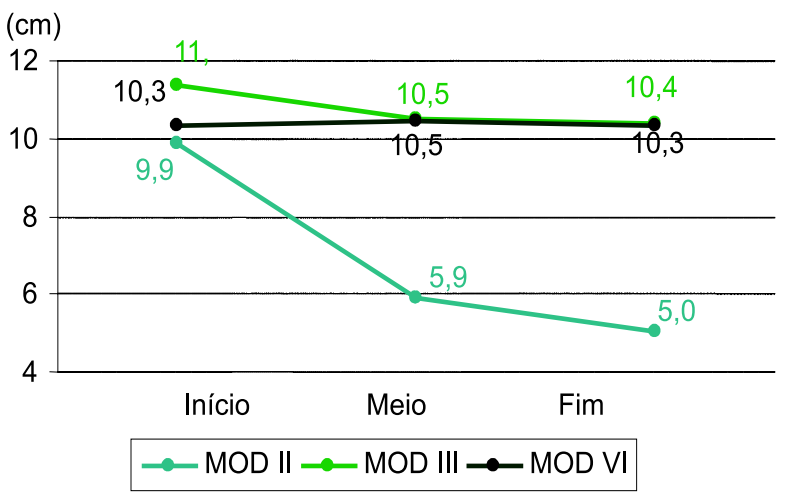

Figura 3 - Valores médios da altura de corte nos módulos II, III e VI.

Figure 3 -Mean values for cut height in modules II, III and VI.

(\%)

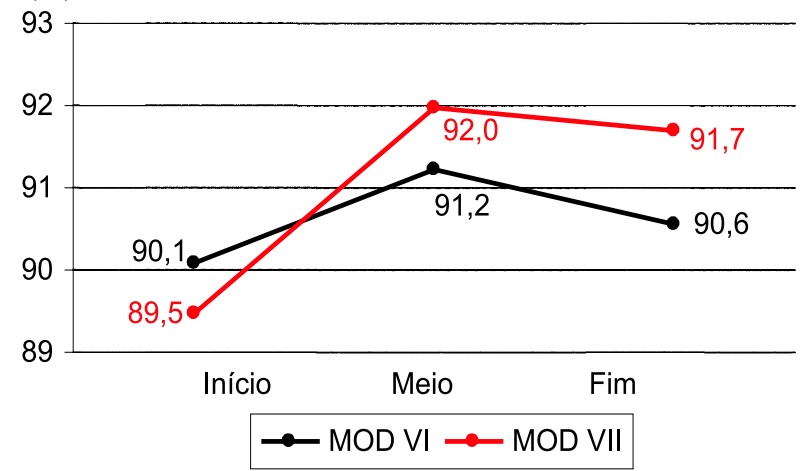

Figura 4 - Valores médios da processamento nos módulos VI e VII.

Figure 4-Mean values for processing in modules VI and VII.

R. Árvore, Viçosa-MG, v.32, n.2, p.291-298, 2008 
$(\%)$

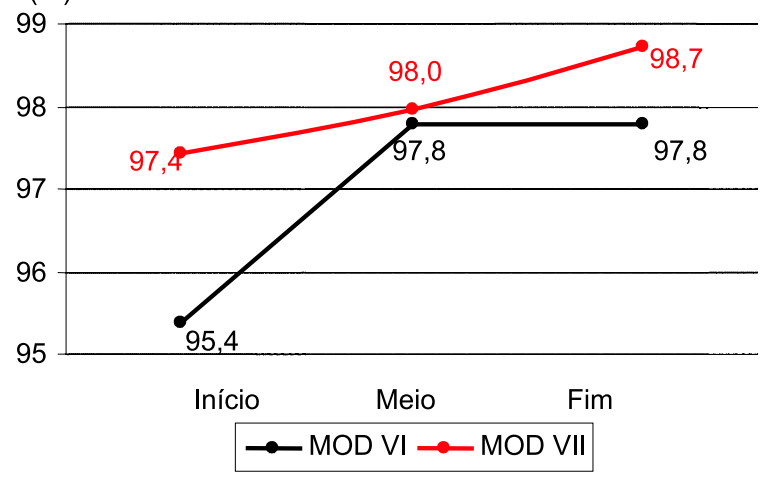

Figura 5 - Desempenho médio da variável eficiência do empilhamento nos módulos VI e VII.

Figure 5 -Average performance of the variable piling up efficiency, in modules VI and VII.

\subsubsection{Produtividade}

A produtividade é a variável que mostra o desempenho final do operador no treinamento, pois contempla simultaneamente todas as variáveis da operação. É importante ressaltar que, nos treinamentos com simulador virtual, os aspectos de qualidade são muito exigidos, sendo as melhorias sempre alcançadas. As médias obtidas no módulo VI foram de 25,9;32,4; e $34,1 \mathrm{~m}^{3} \mathrm{~h}^{-1}$ no início, meio e fim do treinamento, respectivamente, com ganho médio de $31,5 \%$, enquanto no módulo VII as médias foram de 22,7;31,0; e 34,1 $\mathrm{m}^{3} \mathrm{~h}^{-1}$, com ganho médio no desempenho de $51,5 \%$.

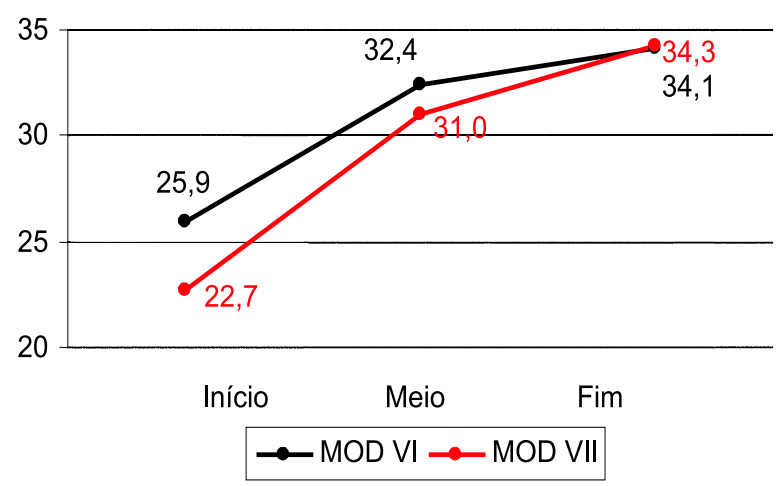

Figura 6 - Valores médios da produtividade nos módulos VI e VII.

Figure 6-Mean values for productivity in modules VI and VII.

R. Árvore, Viçosa-MG, v.32, n.2, p.291-298, 2008
Deve-se observar ainda que a maioria dos operadores obteve desempenho abaixo da meta estabelecida (Tabela 3). Entretanto, tal fato não reflete necessariamente em baixo desempenho, pois as metas são estabelecidas visando sempre estimular os operadores.

A partir das análises realizadas, pôde-se verificar que os maiores ganhos de desempenho dos operadores ocorreram na variável produtividade, seguida por tempo de execução, direção de queda e eficiência no empilhamento. Foi possível ainda verificar que, independentemente da variável analisada, os ganhos de desempenho diminuíram com o decorrer do treinamento, onde os operadores atingiram uma estabilização no aprendizado, mostrando, portanto, que o tempo de duração do treinamento é suficiente para o aprendizado.

\subsection{Análise Estatística}

$\mathrm{Na}$ Tabela 4, apresenta-se o resultado do teste de média das diferentes variáveis e módulos operacionais, realizado pelo teste de Tukey a $5 \%$ de probabilidade. Como pode ser visto, em relação ao tempo de execução houve diferença significativa do primeiro período em relação aos demais em todos os módulos, indicando que os operadores obtiveram melhorias iniciais significativas em seu desempenho.

Em relação à direção de queda, houve diferença significativa no desempenho dos operadores entre os períodos inicial e final de treinamento. Quanto à variável altura de corte, as diferenças ocorreram no período inicial em relação aos demais períodos apenas nos módulos II e III, que se reportam ao posicionamento do cabeçote em diferentes posições. A partir do módulo IV, o operador realizava a derrubada da árvore posicionando o cabeçote próximo ao solo, para minimizar a altura do toco, sendo essa uma operação de difícil melhoria e que levava à obtenção de resultados estatisticamente iguais.

Quanto à eficiência do processamento, observouse diferença significativa nos módulos IV e VII, sendo os demais estatisticamente iguais. Em relação à eficiência no empilhamento, ocorreu essa diferença em todos os módulos, enquanto na variável produtividade se observou diferença significativa nos módulos avaliados, comprovando que houve evolução no desempenho dos operadores no decorrer do treinamento. 
Tabela 3 - Ganhos porcentuais de desempenho dos operadores em relação ao início do treinamento Table 3 - Percent performance gains of the operators in relation to the beginning of the training

\begin{tabular}{|c|c|c|c|c|c|c|c|}
\hline \multirow[t]{2}{*}{ Variável } & \multicolumn{7}{|c|}{ Módulos } \\
\hline & II & III & IV & $\mathrm{V}$ & VI & VII & Média \\
\hline Tempo de execução (s) & 52,0 & 34,4 & 44,1 & 43,7 & 25,0 & 33,4 & 38,8 \\
\hline Direção de queda (graus) & 47,1 & 25,2 & & & & & 36,1 \\
\hline Altura de corte $(\mathrm{cm})$ & 49,4 & 8,8 & 1,7 & 0,0 & 0,1 & & 2,6 \\
\hline Eficiência no processamento $(\%)$ & & & 11,5 & 1,5 & 0,5 & 2,5 & 4,0 \\
\hline Eficiência no empilhamento (\%) & & & & 4,8 & 2,5 & 1,3 & 2,9 \\
\hline Produtividade $\left(\mathrm{m}^{3} / \mathrm{h}\right)$ & & & & & 31,5 & 51,1 & 41,3 \\
\hline
\end{tabular}

Tabela 4 - Teste de médias dos diferentes módulos Table 4 - Test of the different module means

\begin{tabular}{|c|c|c|c|c|c|c|c|}
\hline Módulo & Tratamento & $\begin{array}{l}\text { Tempo } \\
\text { (s) }\end{array}$ & $\begin{array}{c}\text { Direção Queda } \\
\text { (graus) }\end{array}$ & $\begin{array}{l}\text { Altura Corte } \\
(\mathrm{cm})\end{array}$ & $\begin{array}{c}\text { Efic. Proces. } \\
(\%)\end{array}$ & $\begin{array}{c}\text { Efic. Empil. } \\
(\%)\end{array}$ & $\begin{array}{l}\text { Prod. } \\
\left(\mathrm{m}^{3} \mathrm{~h}^{-1}\right)\end{array}$ \\
\hline \multirow[t]{3}{*}{ II } & Início & 68,5 a & $12,6 \mathrm{a}$ & 9,9 a & & & \\
\hline & Meio & $40,2 \mathrm{~b}$ & $7,9 \mathrm{~b}$ & $5,9 \mathrm{~b}$ & & & \\
\hline & Fim & $32,8 \mathrm{~b}$ & $6,7 \mathrm{~b}$ & $5,0 \mathrm{~b}$ & & & \\
\hline \multirow[t]{3}{*}{ III } & Início & $40,6 \mathrm{a}$ & 9,5 a & $11,4 \mathrm{a}$ & & & \\
\hline & Meio & $33,6 \mathrm{ab}$ & $7,8 \mathrm{ab}$ & $10,5 \mathrm{~b}$ & & & \\
\hline & Fim & $26,6 \mathrm{~b}$ & $7,1 \mathrm{~b}$ & $10,4 \mathrm{~b}$ & & & \\
\hline \multirow[t]{3}{*}{ IV } & Início & 82,8 a & & $10,5 \mathrm{a}$ & 82,4 a & & \\
\hline & Meio & $50,8 \mathrm{ab}$ & & $10,4 \mathrm{a}$ & $90,9 \mathrm{~b}$ & & \\
\hline & Fim & $46,3 \mathrm{~b}$ & & $10,3 \mathrm{a}$ & $91,8 \mathrm{~b}$ & & \\
\hline \multirow[t]{3}{*}{$\mathrm{V}$} & Início & 83,9 a & & $10,4 \mathrm{a}$ & 89,9 a & $93,5 \mathrm{a}$ & \\
\hline & Meio & $53,5 \mathrm{~b}$ & & $10,3 \mathrm{a}$ & 91,2 a & $97,1 \mathrm{a}$ & \\
\hline & Fim & $47,2 \mathrm{~b}$ & & $10,4 \mathrm{a}$ & $91,3 \mathrm{a}$ & $98,0 \mathrm{~b}$ & \\
\hline \multirow[t]{3}{*}{ VI } & Início & 123,5 a & & $10,3 \mathrm{a}$ & 90,1 a & 95,4 a & 25,9 a \\
\hline & Meio & $100,1 \mathrm{~b}$ & & $10,5 \mathrm{a}$ & $91,2 \mathrm{a}$ & $97,8 \mathrm{~b}$ & $32,4 \mathrm{ab}$ \\
\hline & Fim & $93,0 \mathrm{~b}$ & & $10,3 \mathrm{a}$ & $90,6 \mathrm{a}$ & $97,8 \mathrm{~b}$ & $34,1 \mathrm{~b}$ \\
\hline \multirow[t]{3}{*}{ VII } & Início & 143,4 a & & & 89,2 a & 97,4 a & 22,7 a \\
\hline & Meio & $111,0 \mathrm{~b}$ & & & $91,9 \mathrm{~b}$ & $98,0 \mathrm{ab}$ & $31,0 \mathrm{~b}$ \\
\hline & Fim & $100,9 \mathrm{~b}$ & & & $91,6 \mathrm{~b}$ & $98,7 \mathrm{~b}$ & $34,3 \mathrm{~b}$ \\
\hline
\end{tabular}

Médias seguidas pela mesma letra são estatisticamente iguais, pelo teste de Tukey a 5\% de probabilidade.

\section{CONCLUSÃO}

Depois da análise e discussão dos resultados, esta pesquisa permitiu a conclusão de que:

a) O treinamento dos operadores com uso de simuladores de realidade virtual permitiu reduzir o tempo de aprendizado, sendo o melhor desempenho adquirido no início do treinamento.

b) Para todas as variáveis analisadas, houve melhorias significativas no desempenho dos operadores no decorrer do treinamento, sendo maiores com relação as variáveis direção de queda, tempo de execução e produtividade.

c) A carga horária de treinamento de $40 \mathrm{~h}$ no simulador virtual mostrou ser suficiente para obtenção de resultados satisfatórios de desempenho, conforme as metas de treinamento.

d) O simulador de realidade virtual mostrou ser uma ferramenta de treinamento de grande eficiência, permitindo a formação de um operador em menor tempo e com redução de custos. Entretanto, o pós-treinamento dos operadores na máquina em situação real de campo é imprescindível para a completa formação dos operadores.

\section{REFERÊNCIAS}

AMABILINI, V. D. Utilização do Harvester na exploração florestal. In: SIMPÓSIO BRASILEIRO SOBRE EXPLORAÇÃO ETRANSPORTE

FLORESTAL, 1., 1991, Belo Horizonte. Anais... Belo Horizonte: 1991.p.349-364.

R. Árvore, Viçosa-MG, v.32, n.2, p.291-298, 2008 
BRAMUCCI, M.; SEIXAS, F. Determinação e quantificação de fatores de influência sobre a produtividade de harvester na colheita florestal. Scientia Forestalis, n.62, p.62-74, 2002.

BRAMUCCI, M. Determinação e quantificação de fatores de influencia sobre a produtividade de "Harvester" na colheita florestal de madeira. 2001. FOLHAS. Dissertação (Mestrado Recursos Florestais) - Escola Superior de Agricultura Luiz de Queiroz, Piracicaba,2001.

CANTO, J. L. et al. Colheita e transporte florestal em propriedades rurais fomentadas no estado do Espírito Santo. Revista Árvore, v.30, n.6, p.981-988, 2006.

LACERDA, J. F. S. B.; MAZON, NOME DO AUTOR. Uso de simuladores de realidade virtual no treinamento de operadores na colheita e transporte florestal. In: SEMINARIO DE ATUALIZAÇÃO SOBRE SISTEMAS DE COLHEITA DE MADEIRAE TRANSPORTE FLORESTAL, 12., 2002, Curitiba. Anais... Curitiba: 2002. p.133-146.2002.
MACHADO, C. C. (Coord.) Colheita

florestal. Viçosa, MG: Universidade Federal de Viçosa, 468 p.2002.

MOREIRA, F. M. T.et al. Avaliação operacional e econômica do "Feller-Buncher" em dois sistemas de colheita de florestas de eucalipto. Revista Árvore, v.28, n.2, p.199-205, 2004.

PACKALÉN, A. Swedish study on harvester simulator training: costs cut, quality maintained. International Forestry Magazine Timberjack News, n.3, p.20-21, 2001.

PARISE, D.; MALINOVSKI, J. R. Análise e reflexões sobre o desenvolvimento tecnológico da colheita florestal no Brasil. In: SEMINÁRIO DE ATUALIZAÇÃO SOBRE SISTEMAS DE COLHEITA DE MADEIRA E TRANSPORTE FLORESTAL, 12., 2002, Curitiba. Anais... Curitiba: 2002. p.78-109. 